\title{
Building Trust: A Cross-Cultural Narrative Process
}

\author{
Yordanka Berg Blanc, William Tilmouth, Chris Perry, Curtis Haines, \\ Ricky Mentha, and Elaine Dietsch
}

\begin{abstract}
The transition to fatherhood is a complex journey scarcely researched in Australian First Nations populations. Historical and political legacy, along with cultural traditions must be taken into consideration before exploring the experiences of Australia's First Nations expectant fathers, especially when the experience is related to the millennia honored "women's business." This article shares the challenges, opportunities, and rewards the authors experienced while researching with Australia's First Nations men who supported their partners during childbirth in a hospital setting. Ethical insights and input from all members of the research team ensured that culturally safe $s$ trategies were used to address all encountered difficulties. Building a trusting relationship with the local community was fundamental to the legitimacy, richness, and success of this research project. Lessons learned from this experience can provide an insight to nonindigenous researchers choosing to conduct or support research with Australia's First Nations people in a respectful and meaningful manner.
\end{abstract}

KEYWORDS: Australian aboriginal and Torres strait islander men; women's health; men's health; First Nations; parenting; cross-cultural collaboration

\section{INTRODUCTION}

We pay respect to the past, present, and future Arrernte people, on whose land this research was undertaken. We acknowledge the loss of lands, cultures and treasures that have had immense consequences for people, communities, and the Arrernte nation. We look forward to continuing to journey together and to a time of historical truth telling, better listening to the voice of all First Nations peoples, and an established treaty.

The transition to fatherhood is a complex and multidimensional journey scarcely researched in First Nations populations (Berg Blanc \& Dietsch, 2018). While qualitative inquiry can achieve outcomes that positively influence the journey to parenthood of Australia's First Nations expectant fathers, it is increasingly important for nonindigenous researchers to critically reflect on their own research practice as well as unforeseen challenges that can emerge during the research process. Informed by Gray and Oprescu (2015), Keikelame and Swartz (2019), and Young et al. (2019), we argue that researchers need to scrutinize and reflect on the selected methodologies used in all stages of the research journey. Of even greater importance, is the need to work with a team of respected First Nations Elders and Matriarchs who are prepared to advise and critique, in order to ensure that every step of the research process is undertaken in a culturally safe and ethically sound manner.

The term First Nations is used throughout this article to acknowledge that Australian Aboriginal and Torres Strait Islander people have been on the continent for many tens of thousands of years prior to European colonization (Sovereign Union, 2016). "Women's business" conveys the uniqueness of women's roles, of ceremonies and lore sacred to Australia's First Nations women (Barclay et al., 1989). 
In this article we highlight the challenges, rewards, and lessons learned before, during, and after the qualitative research was undertaken with Australia's First Nations expectant fathers who were supporting their partners while birthing in hospital. Insights and input from the partnering Elders and Matriarchs in the Arrernte community (the First Nation's people group where the research was sited), anthropologists, local First Nation's researchers, and leaders; were crucial to address the ethical and methodological dilemmas that emerged before, during, and after the research process.

\section{STUDY BACKGROUND}

The research was undertaken in the maternity unit of the major regional hospital in the Arrernte Nation in Central Australia and described the experiences of First Nations men while supporting their partners during labor and birth. Everyone on the research team was acutely aware that this research related to the millennia honored "women's business." However, the research was considered necessary because more First Nations pregnant women are requesting their partner's companionship in the birthing room and occasionally at antenatal care visits (Carter et al., 2004; Reibel et al., 2015; Simmonds et al., 2012). However, little is known about how midwives can best support Australia's First Nations men in this context. As a result, many midwives and other maternity care professionals are unable to meet the culturally unique requirements of these men, their partners, and families.

As a female nonindigenous principal researcher, it was fundamental to scrutinize the relevance of the draft research proposal. The draft research proposal included First Nations pregnant women accessing the maternity services and was discussed with First Nations community leaders before formally proposing the research and seeking ethics committee approval. All those consulted were respected members of the community with strong community ties.

The first and most important step taken, prior to making the final decision to undertake the project, was to discuss the proposed research with the community Matriarchs. Matriarchs from within the Arrernte community were consulted and gave their verbal and tacit approval for the research project which was to explore the experiences of men in what has traditionally been valued as "women's business." During story telling with the principal researcher, they reflected on traditional ways of supporting birthing women "on country" (prior to colonization which gave women no choice but to birth in foreign institutions). These traditional grandmothers also challenged the changes in culture and wondered if the current presence of men in the birthing room might be a contributing factor for postpartum complications in First Nations women coming to birth in the hospital maternity unit. Other questions they wanted answered in future research, included why some women want their partners to be present in the birthing room.

After the Arrernte matriarchs gave their approval, Institutional Ethics Approval was sought and received from the Central Australian Human Research Ethics Committee (HREC-16-447). Ethical approval was also provided by the Executive Director of Medical and Clinical Services and the Maternity Unit Manager of the hospital. The chairperson of the ethics committee was informed about the necessary changes that took place during the research process, which are outlined in this article.

\section{The Research Team}

Fundamental to the research was the preexisting social interaction of the principal researcher with members of the local community who subsequently formed the research team. To effectively engage in this cross-cultural project it was important to access unfamiliar spaces in order to request conversations with key people to enable the principal researcher to better understand the First Nations context and nuances that are significant to ensure the research was at all times culturally respectful and safe for the participants and their communities.

The core research team and authorship of this article included YBB (principal researcher) and WT (Elder and leader of the Central Australia Aboriginal Congress Board of Directors whose mentoring role was to provide ongoing support and cultural insight into the research process). WT facilitated meetings between the principal researcher and First Nations organizations in the community, including the traditional grandmothers (Matriarchs) at the Healing Center and the male Elders at the Central Australia Aboriginal Congress. CP, an anthropologist, guided and assisted the principal researcher through reflective and culturally safe research practice using an anthropological lens. A First Nations registered midwife (CB), a First Nations health practitioner (SK), and two First Nations liaison officers (CD and $\mathrm{CH}$ ) provided invaluable support and advice during the early stages of the investigation that included creation of 
the research proposal, ethical approval, and initial data collection. RM, a Senior Aboriginal researcher, advised the principal researcher about local customs, norms, cultural values, beliefs, and protocols which needed to be followed and how to conduct the research effectively especially when some proposed research methods proved impractical. ED, an academic mentor, provided advice on strategies about how to best communicate and disseminate research findings and how to write for publication.

Unforeseen obstacles encountered during the research process influenced the research team dynamics and interactions. These obstacles included obtaining written consent from the participants, engaging the Aboriginal Liaison Officers (ALOs) in the interview process, and participant's decline to have the interview audiotaped.

Input of all research team members helped to reiterate, refine, and comprehensively implement safe and effective strategies. The challenges experienced throughout the research project corroborated Khankeh et al. (2015) view that a qualitative approach to research does not promise a clear or direct method to address a research problem. A study that was proposed to be small and straightforward developed into a community activity which added significant value to the project but was something not planned or anticipated when the research was originally proposed.

Fitzpatrick et al. (2019), Gray and Oprescu (2015), and Young et al. (2019) argue that fostering partnerships among nonindigenous academic organizations and indigenous health researchers can positively impact the research process. This positive impact translates into better health and wellbeing for First Nations people. Importantly, articulating trusting relationships between First Nations and nonindigenous health professionals within a powerful institutional setting can challenge colonial practices and power imbalances (Ewen et al., 2019; Gray \& Oprescu, 2015). Such challenges were deemed essential in this project, to ensure culturally safe and ethical research practices were always adhered to.

The entire research team ensured that the research was designed in the interests of First Nations people, safeguarded First Nations protocols, and safeguarded the rights of the potential participants. They also provided advice about the research processes. Concepts such as data management, the informed consent process, the interview guide, and how to deal with issues of confidentiality were discussed before starting the study. Nevertheless, potential challenges we would encounter were not anticipated in our early discussions.
It was acknowledged that the research was being conducted in a context (hospital maternity unit) that empowers and privileges western scientific knowledge over the indigenous way of doing, being, and knowing. Therefore, the need to fuse indigenous knowledge and western methodologies in a manner where one was not superior over the other was paramount. Likewise, working within a team of First Nations healthcare workers that included a First Nations registered midwife, a First Nations health practitioner and two First Nations liaison officers, presented both unique research opportunities and unforeseen challenges. In keeping with the arguments of Gray and Oprescu (2015), Keikelame and Swartz (2019), and Toombs (2016), we considered that as nonindigenous and indigenous researchers, it was essential to openly share our experience to encourage all future researchers to take into consideration western approaches that can hinder the transparency of the research process with First Nations people.

The research team provided advice on noncoercive participant recruitment strategies and recommended that face-to-face interviews, originally with an indigenous male research team member present, would be the optimal method of data collection. The team agreed that it would be acceptable to ask the participants if they would like to have the interviews recorded for transcription purposes.

\section{THE RESEARCH JOURNEY}

In compliance with the National Statement on Ethical Conduct in Human Research (2007), the research aims, pathway, confidentiality, benefits of the investigation, and possible risks were explained to each potential participant. This process was designed to ensure potential participants would give their informed consent or informed refusal to participate. The details outlined in the consent form were read to each potential participant. However, all participants who verbally consented to be part of the study declined to sign the consent form. Consequently, the voluntary nature of the study was reemphasized. With the participant's expressed permission, they then gave their verbal consent in the presence of a second midwife.

\section{Obtaining Written Consent}

On reflection, written consent might have been intimidating and confusing for research participants. Aguila 
et al. (2016) found similar challenges while gaining informed consent from a Mexican First Nations Elders cohort. The history of informed consent abuse that caused distrust, exploitation, and dispossession in this population, demanded a better approach from the researchers (Aguila et al., 2016). Additionally, Liamputtong (2008) has argued the assurance of being unidentified is devalued by the participant's need to sign consent forms which might cause doubts about the confidential nature of the research process. Ethical dilemmas related to the informed consent process became apparent. Ensuring evidence of informed consent was very important to the western researchers. However, as Aguila et al. (2016) also found, there can be tension between conducting a culturally appropriate consenting process and producing evidence such as a signed consent form that participants have been recruited voluntarily.

A study conducted by Guillemin et al. (2016) examined the terms in which Australia's First Nations participants agreed to take part in a research project. They concluded that the information that First Nations participants gathered, by talking with and observing the researcher well before the research started, was more valuable to them than the typical Participant Information Sheet they were given to sign later. Guillemin et al. (2016) concluded that by taking this kind of decisionmaking process into account, a more active model of informed consent can be designed and proposed.

From the experience gained during the consent process, it is recommended that the experience of research participants be considered, as well as the diversity and uniqueness of Australia's First Nations men. Researchers need to be flexible in how consent is gained and recorded. In keeping with the arguments of AQ1 Guillemin et al. (2016), Laycock et al. (2012), and Young et al. (2019), these considerations can lead to the use of effective strategies that can benefit the research participants while maintaining the scientific rigor of the study.

Researcher assertions that all collected data will be treated confidentially may not be enough reassurance due to previous abuses of power experienced by First Nations participants. It is strongly recommended to communicate to participants in ways that can easily be understood leaving research jargon aside (Aguila et al., 2016; Fitzpatrick et al., 2019; Guillemin et al., 2016). In line with Liamputtong (2008) and Fitzpatrick et al. (2016, 2019), time needs to be invested to ensure that potential First Nations participants fully understand the information provided to them about the research study and its implications.
In this study, once fully informed consent was assured, arrangements were made to start the semistructured conversational interviews, which were to be conducted face to face with each of the participants. According to the literature, in Australian First Nations culture, it is challenging for a female researcher to interview male participants, as it is considered culturally inappropriate, and it could negatively impact on the openness of stories shared (Aveling, 2013; Jalla \& Hayden, 2014). Accordingly, it was initially planned that one of the two male ALOs would be present during interviews to ensure that the interviews were conducted in a culturally appropriate manner. Their multilingual proficiency could also minimize language barriers and enhance the quality of the communication, as English might not be the first language of the participants. However, this strategy proved to be unacceptable to the participants.

\section{Engagement of ALOs in Interviews}

The first five identified participants accepted the invitation and expressed their desire to be part of the study, but then declined to be interviewed once the ALOs were introduced to them. These participants were not comfortable disclosing their personal experiences to the ALOs and pleaded "shame" as their sole reason. This word, shame, has been interpreted by Simmond A $_{2}$ et al. (2012) and Malcolm and Grote (2007) as the feeling of embarrassment to disclose private issues, especially to an unknown person.

Similarly, Louth (2017) claims that feelings of shame can totally overwhelm and disempower potential research participants. Thus, the presence of the ALOs during interviews posed a significant and unexpected ethical dilemma. The decision of the first five participants who declined to be interviewed was respected and they took no further part in the research. However, invaluable lessons were learned from them. The next five potential participants gave their fully informed consent to participate, without the ALOs being present in the interview and were included in the research.

It is possible that the participants may have considered the liaison officers as strangers from a different clan and with different kinship ties. The Central Australian First Nations community is vast and diverse. This diversity is largely related to geographic, languages and kinship structures, cultural heritage, and knowledge (Welch, 2017). These substantial differences could have affected the progression of the research project, particularly the 
inclusion of the ALOs if the participants considered them strangers. However, if the participants did know the ALOs, that was problematic too as the participants then had greater concerns relating to the anonymity and confidentiality of the information they wished to share. In later team discussions, the importance of considering kinship responsibilities was stressed by the ALOs as was the possibility they might be immediate or extended family members to potential and/or actual participants. The ALOs believed this relationship probably negatively influenced the participants' comfort with being interviewed.

AQ3

According to Simmonds (2002) avoidance relationship exists in Australia's First Nations societal structure with variations within language groups. It was also suggested by other team members that the ALOs might be perceived as hospital authorities whose role was to accomplish the goals of the institution. On later reflection, it is possible that the participants may have either feared being misjudged by others because of their and their partner's desire to be involved in childbirth, an event traditionally considered women's business, or they may have been concerned about the unknown implications their feedback might create.

In contrast, all participants requested their partners be present during the interview. This constituted another dilemma and the ALOs expressed their concern to the principal researcher. It was considered, by the ALOs, culturally inappropriate for them to be in an interview while the participant's partner was present. These unforeseen circumstances discouraged the further direct involvement of the two ALOs in interviews. At the request of all participants and their partners, interviews were conducted by the principal researcher with both the participant and their partner present.

The Institutional Ethics Committee was advised of the proposed amendments to the research process and documented approval to continue with the project was given. Recommendations made by the research team and leaders in the community also encouraged the continuation of the study as they believed it was important to learn from participants in order to find optimal ways to support First Nations expectant fathers in the future.

The input of the research team into amending culturally appropriate recruitment and data collection procedures became even more pivotal as this ensured that the project remained culturally and ethically safe. Without exception the research team members wanted the research to continue and be embraced.

First Nations expectant fathers had been asked if they would feel comfortable talking to a female researcher and surprisingly, this offer was well accepted. This might be due to the preexisting and continuity partnership between the First Nations pregnant woman, her partner, their extended family, and their midwife (principal researcher), during the women's pregnancy, well before this research started.

It is advised that researchers develop their interpersonal skills and consult issues of concern with members of the First Nations community (Fitzpatrick et al., 2019; Jalla \& Hayden, 2014). According to Mapp (2008) and Doody and Noonan (2013) having good interpersonal skills is vital in achieving the ideal balance between being ethically and culturally appropriate, which can lead to successful recruitment and engagement with participants.

\section{Participant Decline to have Interviews Audiotaped}

Although consent to record the interviews was sought by guaranteeing confidentiality and explaining how the individual comments would be de-identified, all participants declined to be recorded. Gray and Oprescu (2015), Jalla and Hayden (2014), and Laycock et al. (2011), suggest that Australia's First Nations pastexperience of involvement in research and the inappropriate use of data may have contributed to an underlying level of distrust First Nations people have toward researchers and research institutions. Additionally, Sim and Wright (2000) argue that participants may feel concerned about their recorded accounts being misinterpreted in their absence and that interviewees may feel that once their voices have been recorded they are indestructible. In the context of this research, interviewees were concerned about speaking of such private topics while being recorded.

While Mapp (2008) claims that the audio recording of interviews is the best choice to collect data, others (e.g., Doody \& Noonan, 2013; Pelto, 2016) acknowledge that in particular situations, handwritten notes are more suitable than audio-recordings as participants preferences must take preference. Pelto (2016) asserts that taping interviews does not absolutely guarantee excellent analysis and research quality. Hence, participants should not feel compelled to be recorded as this can compromise the accounts the researcher gathers (Pelto, 2016).

As a response to the concern raised by all participants, handwritten notes were taken, with their permission during the interviews. This methodological modification worked best in helping the participants to 
feel relaxed and safe in sharing their private and personal experiences with the principal researcher. The process of taking notes was an advantage in this research as traditionally, in Australia's First Nations culture it is inappropriate to establish eye contact during a conversation (Queensland Health, 2015). Likewise, moments of silence during the conversation also aided the data collection process. According to Brédart et al. (2014) and Queensland Health (2015), silence on the part of the interviewee, should not be rushed as it can give the interviewee time to reflect upon the question asked.

It is also recommended that transcripts and notes taken during the interview be verified with the participants to enhance the validity of the collected data and analysis. This strategy can reveal issues that were not spotted during the interview giving additional insight into the findings and the research method used (Abalos et al., 2016). All information obtained from the research interviews were validated by the participants the day following the conversation as a means of member checking to enhance the credibility of the data and to ensure that the participants' experience was correctly interpreted.

This current article has illustrated how western research methodologies can significantly differ from the realities faced undertaking research with Australia's First Nations people in a hospital setting. All modifications made during the research process were crucial in undertaking culturally safe research with Australia's First Nations expectant fathers (Berg Blanc \& Dietsch, 2018). One of the strengths of this qualitative inquiry was being conscious of and motivated to address the challenges encountered during the research process to ensure culturally safe responses to those challenges.

The time allocated for completion of the research project by the academic institution where the principal researcher was completing her degree was another constraint that threatened to limit the quality of the research project. For instance, the time consumed reflecting on the researcher's position within the study, the data collection, and the analysis processes, exceeded the timeframe of the academia. However, the additional time taken added richness to the findings and enabled the necessary changes and interpretations to be made. In line with Young et al. (2019) arguments, researchers need to have the ability to negotiate and adjust the project requirements according to participant's need and feedback, in order to maintain the balance between respectful practice and scientific rigor.

\section{RECOMMENDATIONS}

Research that genuinely responds to the interests and concerns of Australia's First Nations people can help shift power imbalances and enhance the relevance of the research. It is recommended that researchers anticipate and plan the research in a manner that is ethically appropriate, culturally safe and practically feasible, and if needed, be prepared to challenge western academic views and standard protocols during the research journey. Establishing collaborative relationships with First Nations communities and local organizations well before embarking in a research project is paramount to ensuring that all cultural norms are followed. Seeking ongoing consultation and feedback with First Nations leaders in the community, at every phase of the project, enhances the transparency and rigor of the research process. Researchers need to be respectful, humble, honest, and committed, to be able to engage in a research process that can directly benefit the community. First Nations mentorship of western researchers is essential for the holistic, experiential knowledge, and expertise the mentors provide in the design of meaningful research methods.

It would be advantageous if future research could address the questions proposed by the Arrernte Matriarchs and mentioned earlier in this article. These questions include if the presence of men in the birthing room might be a contributing factor for postpartum complications in First Nations women who have birthed in the hospital maternity unit and the reasons why some women want their partners to be present in the birthing room.

\section{CONCLUSION}

This research was a journey of discoveries, challenges, and increasing resilience. Without a doubt, the rewards came not only for the opportunity to learn from each of the study participants but from interactions and developing relationships between the research team members. Research team member roles described in the section on the research team. The trust and genuine partnerships built with First Nations community members over the years positively influenced the outcomes of this research. Engaging Australia's First Nations coresearchers was a unique and rich experience despite the early and 
unexpected withdrawal of the ALOs. All lessons learned can guide nonindigenous researchers to amend Western qualitative methodologies to suit the unique cultural preferences of First Nations people.

\section{REFERENCES}

Abalos, E. E., Rivera, R. Y., Locsin, R. C., \& Schoenhofer, S. O. (2016). Husserlian phenomenology and Colaizzi's method of data analysis: Exemplar in qualitative nursing inquiry using nursing as caring theory. International Journal for Human Caring, 20(1), 19-23. https://doi.org/10.20467/1091-5710-20.1.19

Aguila, E., Weidmer, B. A., Illingworth, A. R., \& Martinez, H. (2016). Culturally competent informed-consent process to evaluate a social policy for older persons with low literacy: The Mexican case. SAGE Open, 6(3). https://doi.org/10.1177/2158244016665886

Aveling, N. (2013). 'Don't talk about what you don't know': On (not) conducting research with/in Indigenous contexts. Critical Studies in Education, 54(2), 203-214. https://doi.org/10.1080/17508487.2012.724021

Barclay, L., Andre, C. A., \& Glover, P. A. (1989). Women's business: The challenge of childbirth. Midwifery, 5(3), 122133. https://doi.org/10.1016/S0266-6138(89)80026-9

Berg, Blanc., Y, \& Dietsch, E. (2018). The experience of Australia's First Nations men supporting their partners during childbirth: A descriptive, interpretive study. International Journal of Childbirth, 8(4), 216-224. https://doi.org/10.1891/2156-5287.8.4.216

Brédart, A., Marrel, A., Abetz-Webb, L., Lasch, K., \& Acquadro, C. (2014). Interviewing to develop Patient-Reported Outcome (PRO) measures for clinical research: Eliciting patients' experience. Health \& Quality of Life Outcomes, 12(1), 1-20. https://doi.org/10.1186/1477-7525-12-15

Carter, E., Lumley, J., Wilson, G., \& Bell, S. (2004). 'Alukura ... for my daughters and their daughters and their daughters. A review of Congress Alukura. Australian and New Zealand Journal of Public Health, 28, 229-234. https://doi.org/10.1111/j.1467-842X.2004.tb00701.x

Doody, O., \& Noonan, M. (2013). Preparing and conducting interviews to collect data. Nurse Researcher, 20(5), 2832. https://doi.org/10.7748/nr2013.05.20.5.28.e32

Ewen, S. C., Ryan, T., \& Platania-Phung, C. (2019). Capacity building of the Australian Aboriginal and Torres Strait Islander health researcher workforce: A narrative review. Human Resources for Health, 17(1), 10. https://doi.org/10.1186/s12960-019-0344-x

Fitzpatrick, E. F. M., Macdonald, G., Martiniuk, A. L., Oscar, J., D’Antoine, H., Carter, M., Lawford, T., \& Elliott,
E. J. (2019). The picture talk project: Aboriginal community input on consent for research. BMC Medical Ethics, 20(1), 12. https://doi.org/10.1186/s12910-019$0349-\mathrm{y}$

Fitzpatrick, E. F. M., Martiniuk, A. L. C., D’Antoine, H., Oscar, J., Carter, M., \& Elliott, E. J. (2016). Seeking consent for research with Indigenous communities: A systematic review. BMC Medical Ethics, 17, 65. https://doi.org/10.1186/s12910-016-0139-8

Gray, M. A., \& Oprescu, F. I. (2015). Role of non-Indigenous researchers in Indigenous health research in Australia: A review of the literature. Australian Health Review, 40, 459-465. https://doi.org/10.1071/AH15103

Guillemin, M., Gillam, L., Barnard, E., Stewart, P., Walker, H., \& Rosenthal, D. (2016). "We're checking them out": Indigenous and non-Indigenous research participants' accounts of deciding to be involved in research. International Journal for Equity in Health, 15(1), 1-10. https://doi.org/10.1186/s12939-016-0301-4

Jalla, C., \& Hayden, G. (2014). Aboriginal health research is not black and white. Lessons from the field. Australian Indigenous Health Bulletin, 14(3), 1-5. http://healthbull etin.org.au/articles/aboriginal-health-research-is-not-b lack-and-whitelessons-from-the-field

Khankeh, H., Ranjbar, M., Khorasani-Zavareh, D., ZarghamBoroujeni, A., \& Johansson, E. (2015). Challenges in conducting qualitative research in health: A conceptual paper. Iranian Journal of Nursing and Midwifery Research, 20(6), 635-641. https://doi.org/10.4103/17359066.170010

Keikelame, M. J., \& Swartz, L. (2019). Decolonising research methodologies: Lessons from a qualitative research project, Cape Town, South Africa. Global Health Action, 12(1), 1561175. https://doi.org/10.1080/16549716.2018.1561175

Laycock, A., Walker, D., Harrison, N., \& Brands, J. (2011). Researching indigenous health: A practical guide for researchers. The Lowitja Institute. https://www.lowitja.o rg.au/researchers-guide.

Liamputtong, P. (2008). Doing research in a cross-cultural context: Methodological and ethical challenges. In E. Diener, W. Glatzer, \& P. Liamputtong (Eds.), Doing cross-cultural research: Ethical and methodological perspectives (pp. 3-21). https://ebookcentral-proquest-com .ezproxy.csu.edu.au

Louth, S. (2017). Indigenous Australians: Shame and respect. In E. Vanderheiden \& C. H. Mayer (Eds.), The value of shame (pp. 187-200). Springer Publishing.

Malcolm, I. G., \& Grote, E. (2007). Aboriginal English: Restructured variety for cultural maintenance. In G. Leitner \& I. G. Malcolm (Eds.), The habitat of Australia's 
Aboriginal languages: Past, present and future (p. 169).

AQ6 https://ebookcentral-proquest-com.ezproxy.csu.edu.au

Mapp, T. (2008). Understanding phenomenology: The lived experience. British Journal of Midwifery, 16(5), 308-311. https://doi.org/10.12968/bjom.2008.16.5.29192

National statement on ethical conduct in human research. (2007). https://www.nhmrc.gov.au/book/national-state AQ7 ment-ethicalconducthuman-research

Pelto, P. J. (2016). Applied ethnography: Guidelines for field research. Routledge.

Queensland Health. (2015). Communicating effectively with Aboriginal and Torres Strait Islander people. https://ww w.health.qld.gov.au/_data/assets/pdf_file/0021/151923 /communicating.pdf

Reibel, T., Morrison, L., Griffin, D., Chapman, L., \& Woods, H. (2015). Young Aboriginal women's voices on pregnancy care: Factors encouraging antenatal engagement. Women \& Birth, 28(1), 47-53. https://doi.org/10.1016/j.wombi.2014.10.003

Sim, J., \& Wright, C. (2000). Recording and organizing data from exploratory studies. In J. Sim \& C. Wright (Eds.), Research in health care: Concepts, designs and methods (pp. 143-150). Stanley Thornes Publishers Ltd.

Simmonds, D., West, L., Porter, J., Davies, M., Holland, C., Preston-Thomas, A., O’Rourke, P. K., \& Tangey, A. (2012). The role for support person for Ngaanyatjarra women during pregnancy and birth. Women and Birth, 25, 79-85. https://doi.org/10.1016/j.wombi.2010.12.007

Sovereign Union. (2016). The difference between the "First Australians" and the "First Nations." http://www.nation alunitygovernment.org/content/differencebetween-firs AQ8 t-australians-and-first-nations

Toombs, M. (2016). The intersection between research and ethics, as it applies to indigenous methodologies. Aboriginal and Islander Health Worker Journal, 40, 810. https://search.informit.com.au/documentSummary; $\mathrm{dn}=787558239930275$;res=IELAPA
Welch, D. M. (2017). Social organization. https://www.aborigi nalculture.com.au/social_organisation.html

Young, J., Watson, K., Craigie, L., Neville, J., \& Hunt, J. (2019). Best practice principles for research with Aboriginal and Torres Strait Islander communities in action: Case study of a safe infant sleep strategy. Women and Birth, 32(5), 460-465. https://doi.org/10.1016/j.wombi.2019.06.022

Disclosure. The authors have no relevant financial interest or affiliations with any commercial interests related to the subjects discussed within this article.

Funding. The author(s) received no specific grant or financial support for the research, authorship, and/or publication of this article.

Correspondence regarding this article should be directed to Yordanka Berg Blanc, BN, PG Dip Midwifery, MSc Midwifery, Alice Springs, NT 0871, Australia. E-mail: ybergblanc@gmail.com

Yordanka Berg Blanc, BN, PG Dip Midwifery, MSc Midwifery, Alice Springs Hospital, Alice Springs, NT, Australia.

William Tilmouth, Chairperson, Children's Ground organization, Alice Springs, NT, Australia.

Chris Perry, RN, Anthropologist, Research Support Coordinator, Alice Springs Hospital, Baker Heart and Diabetes Institute Central Australia, Alice Springs, NT, Australia.

Curtis Haines, Aboriginal Liaison Officer, Alice Springs Hospital, The Gap, NT, Australia.

Ricky Mentha, Aboriginal Health Practitioner, MSc of Public Health, Indigenous Research Fellow, Baker Heart and Diabetes Institute Central Australia, Alice Springs, NT, Australia.

Elaine Dietsch, RM PhD, Griffith University, School of Nursing and Midwifery, Nathan, Queensland, Australia.
AQ10 AQ11

AQ12 AQ13 